\title{
Simulation of Optical Imaging Reconnaissance to Ground
}

\author{
Li Wen-wei \\ China Research and Development Academy of Machinery \\ Equipment; Beijing 100089; China
}

\author{
Meng Hong \\ China Research and Development Academy of Machinery \\ Equipment; Beijing 100089; China
}

\author{
Sun Yong \\ China Research and Development \\ Academy of Machinery Equipment; \\ Beijing 100089; China
}

\author{
Li Guang-yun \\ China Research and Development \\ Academy of Machinery Equipment; \\ Beijing 100089; China
}

\author{
Xue Ji-rong \\ China Research and Development \\ Academy of Machinery Equipment; \\ Beijing 100089; China
}

\begin{abstract}
Optical imaging reconnaissance plays an important role in the intelligence and reconnaissance process, especially widely used in UAV reconnaissance and direct fire weapons. Combined with related requirements, a simulation model of the optical imaging reconnaissance process is proposed, including the simulation model of the target imaging reconnaissance, target location and target extraction. Lastly, the simulation model is test, and the results show that the simulation model can reflect the ground optical imaging reconnaissance simulation reasonably.
\end{abstract}

Keywords- Imaging reconnaissance; target location; target extraction; optical imaging

\section{INTRODUCTION}

Optical imaging reconnaissance equipment to ground includes visible light, infrared camera and video equipment. Imaging reconnaissance are two-dimensional reconnaissance basically, optical imaging is two-dimensional high resolution of azimuth and pitch, and two-dimensional reconnaissance can't target location in theory, but for ground targets reconnaissance, if three-dimensional terrain data of the reconnaissance area is exist or the ground can be treated simplified, then the target can be located.

The imaging reconnaissance simulation is based on mathematical model, the process include identify, locate and extract target simulation. Due to there are many battlefield reconnaissance equipments, mathematical simulation method is used to reduce the simulation nodes and experiment cost.

Photographic results and relationship are researched between different combinations of reconnaissance platforms flight altitude, the field of view of the photographic equipment, visibility and target characteristics. The mathematical simulation method provides a more realistic target data source for the information processing simulation also.

Simulation process includes two steps. First is imaging simulation process, the position and attitude parameters of reconnaissance equipment are known, so reconnaissance region also can be calculated, and target is judged whether exists in the region. Second is target extraction simulation process, if there is target in the photographic region, target extraction is necessary, the resolution of the reconnaissance equipment is need to consider in the process of target extraction simulation, the resolution size and detection distance decided whether the target in reconnaissance area is able to detect and identify [1].

\section{A COORDINATE SYSTEM AND TRANSFORMATION}

The earth and reconnaissance equipment coordinate system are used in this article. The definition and conversion relationship between the coordinate systems are intoduced as follows.

(1) The earth system (n system): $O_{0}-x_{0} y_{0} z_{0}$ (East, north and up).

(2) The reconnaissance equipment system (b system): $O-x_{b} y_{b} z_{b}$, the center line of the lens is the y-axis (to the front), the $\mathrm{x}$-axis is in the symmetry plane of the reconnaissance equipment and to the right.

(3) The Transform of the earth system to reconnaissance equipment system

Set there is a point $\mathrm{A}$ in earth system, its coordinate is $\left(x_{1}, y_{1}, z_{1}\right)$ and reconnaissance equipment coordinate is $\left(x_{0}, y_{0}, z_{0}\right)$, attitude angle is $(\phi, \theta, \gamma)$, then the coordinate of point $\mathrm{A}$ in the reconnaissance equipment system is

$$
\left(\begin{array}{l}
x_{b} \\
y_{b} \\
z_{b}
\end{array}\right)=C_{n}^{b}\left(\begin{array}{l}
x_{1}-x_{0} \\
y_{1}-y_{0} \\
z_{1}-z_{0}
\end{array}\right)
$$

Where $C_{n}^{b}=L_{y}(\gamma) L_{x}(\theta) L_{z}(\phi)$

$$
L_{y}(\gamma)=\left(\begin{array}{ccc}
\cos (\gamma) & 0 & \sin (\gamma) \\
0 & 1 & 0 \\
-\sin (\gamma) & 0 & \cos (\gamma)
\end{array}\right)
$$




$$
\begin{aligned}
& L_{x}(\theta)=\left(\begin{array}{ccc}
1 & 0 & 0 \\
0 & \cos (\theta) & \sin (\theta) \\
0 & -\sin (\theta) & \cos (\theta)
\end{array}\right) \\
& L_{z}(\phi)=\left(\begin{array}{ccc}
\cos (\phi) & \sin (\phi) & 0 \\
-\sin (\phi) & \cos (\phi) & 0 \\
0 & 0 & 1
\end{array}\right)
\end{aligned}
$$

\section{TARGET IMAGING RECONNAISSANCE MATHEMATICAL SIMULATION}

Actual simulation don't simulate the imaging process, therefore it does not produce the simulation images. Imaging area is calculated according to the position and attitude of imaging equipment, then depending on the target physical location to determine whether in the imaging area, if so, indicates the target is the image target, otherwise it is not in the image.

Reconnaissance equipment coordinate is $\left(x_{0}, y_{0}, z_{0}\right)$ in the earth system, attitude angle is $(\phi, \theta, \gamma)$, pitch angle of field view is $\alpha_{y}$, azimuth angle of field view is $\alpha_{x}$, the target's coordinate is $\left(\mathrm{X}_{m}, \mathrm{Y}_{m}, \mathrm{Z}_{m}\right)$ are known. Obtain the target's coordinate in the reconnaissance equipment system is

$$
\left(\begin{array}{l}
x_{b} \\
y_{b} \\
z_{b}
\end{array}\right)=C_{n}^{b}\left(\begin{array}{l}
X_{m}-x_{0} \\
Y_{m}-y_{0} \\
Z_{m}-z_{0}
\end{array}\right)
$$

The pitch angle $\alpha_{m y}$ and azimuth angle $\alpha_{m x}$ are calculated in reconnaissance equipment system based on the target's coordinate in the reconnaissance equipment system:

$$
\begin{gathered}
\alpha_{m y}=\arctan \frac{z_{b}}{y_{b}}, \quad \alpha_{m x}=\arctan \frac{x_{b}}{y_{b}} \\
\alpha_{m x} \in\left(-\frac{1}{2} \alpha_{x}, \frac{1}{2} \alpha_{x}\right) \\
\text { If } \alpha_{m y} \in\left(-\frac{1}{2} \alpha_{y}, \frac{1}{2} \alpha_{y}\right) \text { and } y_{b}>0 \text {, the target is in the }
\end{gathered}
$$
reconnaissance region, otherwise not in the reconnaissance region.

\section{TARGET LOCATION SIMULATION [2]}

The target's coordinate can be calculated in the earth system, based on target's coordinate in the reconnaissance equipment system and the attitude of the reconnaissance equipment. And the mean of the Positioning System positioning error is 0 , the intermediate error is $\sigma_{d}$, the mean of the altimeter height measurement error is 0 , the mean square deviation is $\sigma_{z}, \rho$ is the conversion factor between the intermediate error and mean square error, the value is 1.4826 .

The target's coordinate in the reconnaissance equipment system is obtained from formula (2), assume that the error of reconnaissance equipment output posture follows Gaussian white noise distribution, whose mean is 0 and mean square deviation is $\sigma_{\phi}, \sigma_{\theta}, \sigma_{\gamma}$.

$$
\begin{aligned}
& \phi_{u}=\phi+\sigma_{\phi} \text { gauss } \\
& \theta_{u}=\theta+\sigma_{\theta} \text { gauss } \\
& \gamma_{u}=\gamma+\sigma_{\gamma} \text { gauss }
\end{aligned}
$$

The target's coordinate can be obtained in the earth system.

$$
\left(\begin{array}{l}
x_{m} \\
y_{m} \\
z_{m}
\end{array}\right)=C_{b}^{n}\left(\begin{array}{l}
x_{b} \\
y_{b} \\
z_{b}
\end{array}\right)+\left(\begin{array}{l}
x_{\mathrm{d}}^{\prime} \\
y_{\mathrm{d}}^{\prime} \\
z_{\mathrm{d}}^{\prime}
\end{array}\right)
$$

Where $C_{b}^{n}=L_{z}^{T}\left(\gamma_{u}\right) L_{x}^{T}\left(\theta_{u}\right) L_{y}^{T}\left(\phi_{u}\right)$

$$
\left(\begin{array}{c}
x_{\mathrm{d}}^{\prime} \\
y_{\mathrm{d}}^{\prime} \\
z_{\mathrm{d}}^{\prime}
\end{array}\right)=\left(\begin{array}{c}
x_{0}+\rho \cdot \sigma_{d} \cdot \text { gauss } \\
y_{0}+\rho \cdot \sigma_{d} \cdot \text { gauss } \\
z_{0}+\sigma_{z} \cdot \text { gauss }
\end{array}\right)
$$

\section{TARGET EXTRACTION PROCESS SIMULATION}

A. Judge the target could be detected or not [3]

Assume that resolution of the reconnaissance equipment is $\mathrm{M}^{*} \mathrm{~N}$ pixels, the coordinate is $\left(x_{0}, y_{0}, z_{0}\right)$ in the earth system, the attitude angle is $(\phi, \theta, \gamma)$, the level of field view angle is $\alpha_{y}$, the azimuth of field view angle is $\alpha_{x}$, the target's coordinate is $\left(\mathrm{X}_{m}, \mathrm{Y}_{m}, \mathrm{Z}_{m}\right)$ in the earth system, the target length, width, high is $\mathrm{L}, \mathrm{K}, \mathrm{H}$, the length and width of photographs are Lx and Ly respectively. Set the distance between the target and reconnaissance equipment is $\mathrm{R}$, the target equivalent area is $\mathrm{S}$ according to photographic reconnaissance equipment, the target equivalent radius is $\mathrm{r}$ on the photo. After that, R, S, r is calculated respectively.

1) Calculate the distance between the target and reconnaissance equipment

$$
R=\sqrt{\mathrm{X}_{m}{ }^{2}+\mathrm{Y}_{m}{ }^{2}+\mathrm{Z}_{m}{ }^{2}}
$$

2) Calculate the target projection in the photographic observations plane 
If the target attitude angle is known, the projected area on the photographic observation plane could be calculated accurately, and the calculation is complicated, so it is treated simplified. The face area, side area and overlooking area of target is $\mathrm{S} 1, \mathrm{~S} 2$ and $\mathrm{S} 3$ respectively, set

$$
\begin{gathered}
\vec{R}=\left(\mathrm{X}_{m}, \mathrm{Y}_{m}, \mathrm{Z}_{m}\right) \\
\vec{I}_{R}=\vec{R} / R \\
\vec{n}_{1}=\left(\cos \theta_{\mathrm{m}} \cos \phi_{m}, \cos \theta_{\mathrm{m}} \sin \phi_{m}, \sin \theta_{\mathrm{m}}\right)^{T} \\
=\left(\mathrm{v}_{\mathrm{x}}, \mathrm{v}_{\mathrm{y}}, \mathrm{v}_{\mathrm{z}}\right)^{T} \\
\overrightarrow{\mathrm{n}}_{2}^{\prime}=\left(-\mathrm{v}_{\mathrm{y}}, \mathrm{v}_{\mathrm{x}}, 0\right)^{T} / \sqrt{\mathrm{v}_{\mathrm{x}}^{2}+\mathrm{v}_{\mathrm{y}}^{2}} \\
\overrightarrow{\mathrm{n}}_{3}^{\prime}=\vec{n}_{1} \times \overrightarrow{\mathrm{n}}_{2}^{\prime}=\frac{\left(-\mathrm{v}_{\mathrm{x}} \mathrm{v}_{\mathrm{z}}, \mathrm{v}_{\mathrm{y}} \mathrm{v}_{\mathrm{z}}, \mathrm{v}_{\mathrm{x}}^{2}+\mathrm{v}_{\mathrm{y}}^{2}\right)^{T}}{\left(\sqrt{\mathrm{v}_{\mathrm{x}}^{2}+\mathrm{v}_{\mathrm{y}}^{2}}\right)} \\
\vec{n}_{2}=\cos \gamma_{m} \vec{n}_{2}^{\prime}+\sin \gamma_{m} \vec{n}_{3}^{\prime} \\
\vec{n}_{3}=-\sin \gamma_{m} \vec{n}_{2}^{\prime}+\cos \gamma_{m} \vec{n}_{3}^{\prime}
\end{gathered}
$$

The projected area of the target in the observation plane as follows:

$$
S=\left|\vec{I}_{R} \cdot \vec{n}_{1}\right| S_{1}+\left|\vec{I}_{R} \cdot \vec{n}_{2}\right| S_{2}+\left|\vec{I}_{R} \cdot \vec{n}_{3}\right| S_{3}
$$

3) Calculate the target equivalent radius $r$ in the photo by $S$

As shown in below figure, set $\mathrm{O}$ is photographic reconnaissance equipment, $\mathrm{OA}$ is distance $\mathrm{D}$ between reconnaissance equipment and the target, $\mathrm{AB}$ is the equivalent radius $r_{s}$ of the target, $\mathrm{AG}$ is the half amplitude of the photographic region, EC is the half of the photo equivalent radius length, that is $\frac{1}{2} L_{x}$, ED is the target equivalent radius $\mathrm{r}$ in the photo, the target equivalent radius can be obtained by $\mathrm{S}$

$$
r_{s}=\sqrt{S / \pi}
$$

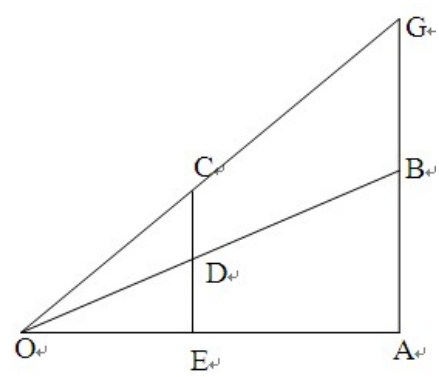

Figure 1. Position relationship
Then $\mathrm{r}$ could be obtained.

$$
r=\frac{r_{s} L_{x}}{2 \operatorname{Dtg}\left(0.5 \alpha_{y}\right)}
$$

The approximate number of pixels is:

$$
n=\frac{r}{L_{x}} M
$$

The target can be detected when $n>=\delta_{n}$, otherwise it can't be detected, $\delta_{n}$ can be obtained through the experimental data.

\section{B. Target extraction accuracy simulation [4,5]}

Suppose the point target extraction error follows the Gaussian distribution, mean is 0 and mean square deviation is $\sigma_{\mathrm{D}}$ (distance error). Suppose the surface target location extraction error follows the Gaussian distribution, mean is 0 and mean square deviation is $\sigma_{\mathrm{D} 1}$ (distance error). The extraction error of long side, short sides and trend follow the Gaussian distribution, mean is 0 , mean square deviation is $\sigma_{\mathrm{L}}$, $\sigma_{\mathrm{L} 1}, \sigma_{\beta}$ respectively. Point target's coordinate after extraction is:

$$
\begin{aligned}
& x^{\prime}=x_{m}+\sigma_{D} \cdot \text { gauss } \\
& y^{\prime}=y_{m}+\sigma_{D} \cdot \text { gauss }
\end{aligned}
$$

Surface target's coordinate after extraction is:

$$
\begin{aligned}
& x^{\prime}=x_{m}+\sigma_{D 1} \cdot \text { gauss } \\
& y^{\prime}=y_{m}+\sigma_{D 1} \cdot \text { gauss }
\end{aligned}
$$

Set $1_{0}, l_{1}$ and $\beta$ are the true values of the long side, short side and trend, the values after extraction are:

$$
\begin{aligned}
& l_{0}^{\prime}=l_{0}+\sigma_{L} \cdot \text { gauss } \\
& l_{1}^{\prime}=l_{1}+\sigma_{L 1} \cdot \text { gauss } \\
& \beta^{\prime}=\beta+\sigma_{\beta} \cdot \text { gauss }
\end{aligned}
$$

\section{The target type and friend-or-foe property simulation}

Set the target type recognition probability of information processing is $\mathrm{P}_{1}$, the friend-or-foe property recognition probability is $\mathrm{P}_{2}$. Extract random number $\mathrm{P}_{1}$ from uniformly distributed interval $[0,1]$, if $\mathrm{P}_{1}^{\prime} \leq \mathrm{P}_{1}$, set the true type of the target as a reconnaissance target type, otherwise, set the closest target in shape as the reconnaissance target types.

Extract random number $\mathrm{P}_{2}$ from uniformly distributed interval [0,1], if $\mathrm{P}_{2} \leq \mathrm{P}_{2}$, set the target friend-or-foe property as the reconnaissance friend-or-foe property. Otherwise, set the contrary property of the objectives true friend-or-foe property as the reconnaissance target friend-or-foe property. 


\section{SIMULATION EXPERIMENT}

Simulation program is established based on the above model, and simulation experiment is carried based on 1:10000 scale topographic data of certain area. 50 targets are placed with discrete form in the test area, the influence of the different performance parameters of reconnaissance equipment and reconnaissance platforms on target reconnaissance results are test and analyzes, the specific results are shows as the following table, simulation results in the table are the average of the 50 target reconnaissance simulation results.

TABLE I. RECONNAISSANCE RESULTS

\begin{tabular}{|c|c|c|c|c|}
\hline \multirow{2}{*}{ Platform state } & Height (m) & 500 & 800 & 1000 \\
\hline & Viewing angle (degree) & 60 & 70 & 90 \\
\hline \multirow{5}{*}{$\begin{array}{c}\text { Reconnaissance } \\
\text { platform performance } \\
\text { parameters }\end{array}$} & Middle position error (m) & 5 & 5 & 5 \\
\hline & $\begin{array}{c}\text { Height measurement } \\
\text { error }(m)\end{array}$ & 1 & 1 & 1 \\
\hline & Azimuth error (mil) & 1 & 1 & 1 \\
\hline & Roll error (mil) & 1 & 1 & 1 \\
\hline & Pitch error (mil) & 1 & 1 & 1 \\
\hline \multirow{3}{*}{$\begin{array}{c}\text { Reconnaissance } \\
\text { equipment } \\
\text { performance } \\
\text { parameters }\end{array}$} & $\begin{array}{c}\text { Azimuth resolution } \\
\text { (pixel) }\end{array}$ & 1000 & 1000 & 1000 \\
\hline & Level resolution (pixel) & 800 & 800 & 800 \\
\hline & $\begin{array}{c}\text { Azimuth field of view } \\
\text { (degree) }\end{array}$ & 45 & 45 & 45 \\
\hline
\end{tabular}

\begin{tabular}{|c|c|l|l|l|}
\hline & $\begin{array}{c}\text { Level field of view } \\
\text { (degree) }\end{array}$ & 35 & 35 & 35 \\
\hline Locate Error & $(\boldsymbol{m})$ & 15.5 & 17.0 & 21.8 \\
\hline Extract Error & $(\mathbf{m})$ & 2.5 & 2.6 & 3.0 \\
\hline $\begin{array}{c}\text { Target type } \\
\text { recognition }\end{array}$ & Correct rate (\%) & 85 & 79 & 75 \\
\hline $\begin{array}{c}\text { Identification friend- } \\
\text { or-foe }\end{array}$ & Correct rate (\%) & 95 & 90 & 88 \\
\hline
\end{tabular}

\section{REFERENCES}

[1] Hu Xiao-yun, "Study on reconnaissance functional simulation model of visible light reconnaissance equipment," Command control and simulation, 2007, vol. 29 (1), pp. 95-97, 102.

[2] Zhang Xiao-hu, Di Hui, Zhou Jian, etc, "A new method for moving target positioning under the condition of monocular camera," Journal of the National University of Defense Technology, 2006, vol. 28 (5), pp. 114-118.

[3] Li Zeng-Lu, "Simulation for the scouting system searching objects," Acta Simulata Systematica Sinica, 2002, vol. 14 (6), pp. 818-820.

[4] Huang He, Lv Ying-jie, "Calculating method and simulation analysis on discovery target probability of optical Imaging satellite," Electro-Optic Technology Application, 2007, vol. 22 (4), pp. 21-23, 37.

[5] Kang Shao-dan, Wang Zhuang; $\mathrm{Hu}$ Wei-dong, "Target fusion identification algorithm based on electronic and optical reconnaissance," Systems Engineering and Electronics, 2004, vol. 26 (6), pp. 589-591, 667. 